\section{Cureus}

\title{
MASTERS-D Study: A Prospective, Multicenter, Pragmatic, Observational, Data-Monitored Trial of Minimally Invasive Fusion to Treat Degenerative Lumbar Disorders, One-Year Follow-Up
}

Jörg Franke ${ }^{1}$, Neil Manson ${ }^{2}$, David Buzek ${ }^{3}$, Arkadiusz Kosmala ${ }^{4}$, Ulrich Hubbe ${ }^{5}$, Wout Rosenberg ${ }^{6}$, Paulo Pereira ${ }^{7}$, Roberto Assietti ${ }^{8}$, Frederic Martens ${ }^{9}$, Khai Lam ${ }^{10}$, Giovanni Barbanti Brodano ${ }^{11}$, Peter Durny ${ }^{12}$, Zvi Lidar ${ }^{13}$, Kai Scheufler ${ }^{14}$, Wolfgang Senker ${ }^{15}$

1. Klinikum Dortmund 2. Horizon Health Network 3. Karvinska hornicka nemocnice 4. Orthopedics, Klinikum Kulmbach 5. Faculty of Medicine, University of Freiburg, Germany, Neurosurgical Clinic, Medical Center, University of Freiburg, Germany 6. Orthopedics, Franciscus Ziekenhuis, Rosendaal 7. Faculty of Medicine of the University of Porto 8. Neurosurgery, Fatebenefratelli Hospital 9. Orthopedics, Ons Lieve Vrouw Ziekenhuis 10. Spine surgery, London Bridge Hospital, London, UK 11. Istituto Ortopedico Rizzoli, Bologna, Italy 12. Neurosurgery, Ustredna vojenska nemocnica SNP, Ruzomberok, Slovakia 13. Neurosurgery, The Tel Aviv Sourasky Medical Center, Tel Aviv, Israel 14. Neurosurgery, Klinikum Dortmund 15. Klinikum Amstetten

$\square$ Corresponding author: Jörg Franke, joerg.franke@klinikumdo.de Disclosures can be found in Additional Information at the end of the article

\section{Abstract}

The objective of the study is to assess effectiveness and safety of minimally invasive lumbar interbody fusion (MILIF) for degenerative lumbar disorders (DLD) in daily surgical practice and follow up with patients for one year after surgery.

A prospective, multicenter, pragmatic, monitored, international outcome study in patients with DLD causing back/leg pain was conducted (19 centers). Two hundred fifty-two patients received standard of care available in the centers. Patients were included if they were aged $>18$ years, required one- or two-level lumbar fusion for DLD, and met the criteria for approved device indications. Primary endpoints: time to first ambulation (TFA) and time to surgery recovery (TSR). Secondary endpoints: patient-reported outcomes (PROs)--back and leg pain (visual analog scale), disability (Oswestry Disability Index (ODI)), health status (EQ-5D), fusion rates, reoperation rates, change in pain medication, rehabilitation, return to work, patient satisfaction, and adverse events (AEs). Experienced surgeons ( $\geqslant 30$ surgeries pre-study) treated patients with DLD by one- or two-level MILIF and patients were evaluated for one

Received 03/13/2016 Review began 03/30/2016 Review ended 06/07/2016 Published 06/13/2016

\section{(c) Copyright 2016}

Franke et al. This is an open access article distributed under the terms of the Creative Commons Attribution License CC-BY 3.0., which permits unrestricted use, distribution, and reproduction in any medium, provided the original author and source are credited. year (NCT01143324).

At one year, 92\% (233/252) of patients remained in the study. Primary outcomes: TFA, $1.3 \pm 0.5$ days and TSR, $3.2 \pm 2.0$ days. Secondary outcomes: Most patients (83.3\%) received one level MILIF; one (two-level) MILIF mean surgery duration, 128 (182) min; fluoroscopy time, 115 (154) sec; blood loss, 164 (233) $\mathrm{mL}$; at one year statistically significant $(\mathrm{P}<.0001)$ and clinically meaningful changes from baseline were reported in all PROs--reduced back pain $(2.9 \pm 2.5 \mathrm{vs}$. $6.2 \pm 2.3$ at intake), reduced leg pain ( $2.2 \pm 2.6$ vs. $5.9 \pm 2.8)$, and ODI $(22.4 \% \pm 18.6$ vs. $45.3 \% \pm$ 15.3), as well as health-related quality of life (EQ-5D index: $0.71 \pm 0.28$ vs. $0.34 \pm 0.32$ ). More of the professional workers were working at one year than those prior to surgery (70.3\% vs. 55.2\%). Three AEs and one serious AE were considered procedure-related; there were no deep site

\section{How to cite this article}

Franke J, Manson N, Buzek D, et al. (June 13, 2016) MASTERS-D Study: A Prospective, Multicenter, 
infections or deaths.

This is the first study evaluating MILIF for treatment of DLD in daily clinical practice. Clinically significant improvements were observed in all endpoints. Short-term post-surgery improvements (four weeks) were maintained through one year with minimal complications. Our results suggest that MILIF has good-to-excellent outcomes for the treatment of DLD in a broad patient population under different clinical conditions and healthcare delivery systems.

Categories: Neurosurgery, Orthopedics

Keywords: minimally invasive lumbar fusion, minimal access spinal technologies, degenerative lumbar disorders, patient-reported outcomes, pragmatic

\section{Introduction}

In the past 25 years, minimally invasive spine surgery (MIS) has emerged as an alternative to traditional open surgery to treat degenerative lumbar diseases (DLD). MIS allows less extensive manipulation of surrounding tissues while accomplishing the same goals and objectives for target tissues [1-2]. Short-term benefits of MIS include reductions in intraoperative blood loss, postoperative pain, approach-related soft tissue damage and duration of hospital stay vs open methods. MIS is also associated with earlier ambulation and an increased proportion of patients returning to work vs. open surgeries [3-5].

Published, long-term MIS studies have been associated with one or more of the following study limitations: low number of patients, surgeries performed at a single site using a single surgical technique, retrospective observational design, variability in reporting and surgeon learning curve [4, 6-13]. While available data suggests decreased morbidity and at least comparable outcomes vs. open surgeries for the immediate and short-term postoperative period [14], there is limited data to support the long-term superiority of MIS over open techniques [3].

A multicenter study reflecting the usual surgical practice was required to observe outcomes and effectiveness of the minimally invasive techniques performed by experienced surgeons.

\section{Purpose of the study}

The objectives of the MASTERS-D study were to observe patients undergoing minimally invasive lumbar interbody fusion (MILIF) short-term recovery of one year to document and evaluate the clinical and radiological patient outcomes in both short-term duration (postoperative, four weeks) [5] and mid-term duration (one year) after MILIF using posterior lumbar interbody fusion (PLIF) or transforaminal lumbar interbody fusion (TLIF) techniques for the treatment of DLDs in a broad patient population.

\section{Materials And Methods}

The study methodology has been described in a previous publication by the authors in Pereira et al [5].

\section{Study environment}

The study was initiated by a group of experienced surgeons and conducted in 19 centers across 14 countries (Europe, Canada, and the Middle East). To avoid a potential learning curve effect, all participating surgeons were required to have performed $\geqslant 30$ pre-study minimally invasive, instrumented lumbar interbody fusion procedures for DLD and surgical indications. Surgical indications, protocols, follow-up length, monitoring, and data analysis were decided before the 
study was initiated. Each surgeon was asked to contribute a maximum of 30 consecutive patients from their clinic at the start of the study, and each clinical center had a research nurse participating for the collection of data. The study group met regularly (quarterly) over the internet to discuss progress, barriers, or problems. The sponsor (Medtronic) of the study participated in the monitoring of the data, but did not participate in the study design or had any input in the analysis of results.

\section{Study design and patients}

MASTERS-D was a prospective, international, observational study with a one-year follow-up and monitored (NCT01143324) patients with DLD causing back/leg pain referred to a surgical practice with experienced surgeons. All patients who met the inclusion criteria and were willing to participate in the study were enrolled consecutively in order to reduce selection bias. All patients received routine standard of care according to hospital protocol to reflect the real life practice circumstances, and all patients signed an informed consent/patient data release form.

\section{Monitoring}

Monitoring visits were conducted throughout the study by monitors from Medtronic and independent professionals from clinical research organizations (CROs). This approach aimed to ensure compliance with protocol, adherence to data collection procedures, accuracy of submitted clinical data, and verify proper maintenance of records for the duration of the study.

\section{Ethics Approval}

Ethics committee (EC), institutional review board (IRB), human research ethics committee (HREC) requirements varied across centers and countries. The study was conducted in compliance with the latest version of the Declaration of Helsinki, and laws and regulations of the countries in which the study was conducted, including data protection laws and the Clinical Investigation Plan. The EC, IRB, HREC, data protection authority, and Competent Authority approvals received were as follows: 1. Klinikum Amstetten, Ethikommission fur das Bundesland Niederosterreich am Sitz des Amtes der NO Landesregierung, Abteilung Sanitats und Krankenanstaltenrecht, Austria; 2. O.L.V.Aalst, Local Ethisch Comite, OL Vrouwziekenhuis Aalst, Belgium; 3. Horizon Health Network, East Spine Center, Local Research Ethics Board, Canada; 4. Universitatsklinikum Freiburg, Central ethics committee (Universität Freiburg, Germany); 5. Mediterraneo Hospital, Local ethics committee, Notification to local authority, submission to Data Protection Authority; 6. Tel Aviv Sourasky Medical center, Local Ethics committee, Tel Aviv Medical Center, Israel and Hospital director; 7. Istituti Ortopedici Rizzoli, Comitato Etico Scientifico, Istituto Rizzoli di Bologna, Italy; 8. Fatebenefratelli Hospital, Comitato Etico Scientifico, Ospedale Fatebenefratelli, Italy; 9. Medical University of Gdansk, Local independent medical ethic committee for scientific research of Gdansk University, Poland; 10. Hospital S Joao, Local comissao de etica do Hospital S Joao, Portugal and Submission to Data Protection Authority; 11. Ustredna vojenska nemocnica SNP, Eticka Komisia, Ustrednex Vojenskej Nomocnice SNP, Slovakia; 12. Hospital Clinic de Barcelona, Comite de investigacion del hospital Clinic de Barcelona, Spain; 13. Guys \& St. Thomas NHS Trust, Local NHS National Ethics Service, UK. The following centers did not require ethics committee approval by a local agency, and investigator statements were collected and confirmed by investigators: 1 . Karvinska hornicka nemocnice, Czech Republic; 2. Klinikum Kulmbach, Germany, 3. Universitatsklinikum Magdeburg, Germany; 4. Marienhaus Klinikum, Germany; 5. Franziskus Ziekenhis Roosendaal, Netherlands; 6. Bergman Clinics, Netherlands; 7. London Bridge Hospital, Ethics Committee.

Eligibility Criteria 


\section{Cureus}

Eligibility criteria were broad and based on the approved indications for the devices used in this study. Inclusion criteria were: eligible patients aged $>18$ years were clinically assessed as requiring a single- or double-level instrumented lumbar fusion for the treatment of the degenerative lumbar spine. All patients were offered to participate in the study and planned to undergo the fusion procedure using PLIF or TLIF techniques to receive a CD Horizon Spinal System (Medtronic Sofamor Danek Inc., Memphis, TN, USA) via the MAST approach (Minimal Access Spinal Technologies, Medtronic Sofamor Danek USA, Inc.) in accordance with the device label. Exclusion criteria were: patients who had previously undergone extensive open lumbar spine surgery other than microdiscectomy were excluded from the study, as were patients with indications for a procedure other than DLD.

\section{Surgical Procedures}

The definitions applied as per study protocol are presented in Table 1 . The muscle sparing, minimally invasive approach could be performed unilaterally or bilaterally for instrumentation and spinal decompression at the surgeon's discretion. One or two cages were placed in the intervertebral space to maintain or restore disc height. To achieve interbody fusion, bone grafts and/or substitutes were used. The posterior stabilization of the treated spinal segments was performed using the CD Horizon Spinal System [5].

\section{Term}

Open lumbar

procedure

Minimally

invasive

procedure

Mini-open

technique

Percutaneous technique

\section{Description}

Surgical technique using a midline approach and requiring a partial or complete detachment of the lumbar fascia and paraspinal muscles to address the spinal pathology and placement of instrumentation

Muscle-sparing surgical technique using an intermuscle- or transmuscle-splitting approach, minimizing detachment of the lumbar fascia and paraspinal muscles to address the spinal pathology and placement of instrumentation

Instrumentation placement using direct vision of target structures via an intermuscle-splitting approach Instrumentation placement using radiographic or navigation guidance via stab incisions without direct vision of target structures

TABLE 1: Surgical techniques and instrumentation definitions for posterior lumbar surgery and instrumentation according to the study protocol

\section{Endpoints and study assessments}

The primary objectives of the study were to observe and document patient short-term recovery after surgery [5] which included: a) time to first ambulation (TFA) (defined as the number of days after surgery before patients were able to get out of bed and ambulate with or without assistance), and b) time to surgery recovery (TSR) (defined as the number of days after surgery until patients no longer needed intravenous infusion of analgesic drugs, had no surgery-related complications/adverse events (AEs) impeding discharge, and no longer needed nursing care).

Secondary outcomes were defined as patient-reported outcomes (PRO) and radiological outcomes one year after surgery; PROs included back and leg pain intensity measured by visual analog scale (VAS) scores [15] Oswestry disability index (ODI) scores [16], and EuroQoL five 
dimension (EQ-5D) scores [17]. Back and leg pain were assessed on a $10 \mathrm{~cm}$ VAS scale $(0=$ no pain, 10 = the worst possible pain) preoperatively, during the hospital stay, at surgery recovery day, at day two after surgery, at discharge, and at all follow-up visits. Disability was rated on a $0 \%$ to $100 \%$ scale preoperatively and postoperatively at all follow-up visits using the ten-item ODI $(0 \%=$ minimal disability, $100 \%$ = maximal disability $)$; ODI was recognized by Mapi Research trust. Health related quality of life was assessed pre-operatively and at all follow-up visits using the five-item EQ-5D questionnaire (if it was part of standard of care at the study center) with three levels for each dimension: no problems, some problems, or extreme problems. EQ-5D index scores were obtained using the UK population value set (http://www.euroqol.org). In addition, patients completed the EQ-VAS to self-rate their overall health state on a 0 to 100 scale $(0=$ maximal health-related problems, $100=$ minimal healthrelated problems).

Clinical and radiological outcomes included the documentation of AEs and fusion rate assessment at one year after surgery. The fusion rate was assessed via computed tomography (CT) scan or X-rays at those sites where this assessment was part of standard of care. As per protocol for assessment by CT, the criteria for fusion was bony bridging, and when assessed through X-rays the criteria were bony bridging, $<4^{\circ}$ motion in flexion/extension views, and integrity of implanted devices. Other clinical endpoints assessed were: documentation of rehabilitation and return to work; reoperation rates, defined as the proportion of patients needing a second intervention at the treated level(s) within one year; proportion of patients needing intervention at an adjacent level; patient satisfaction as assessed by the surgeon; and a change in consumption of pain medication over time compared with baseline. Work status was assessed at the preoperative visit, at 4 weeks and 3, 6, and 12 months postoperatively [11].

Data were collected prospectively by the investigators (surgeons) or the study nurses as per standard of care for each center and included patient medical history, preoperative data, assessment of time from surgery to TFA, TSR and time to discharge. The objective of the TSR day assessment is to record the day when the patient could be discharged based on his actual clinical condition. In some cases the effective day of discharge may be prolonged by factors other than the patient's clinical recovery such as social factors, reimbursement issues, and/or hospital protocol, in these cases the discharge day was not recorded. Postoperative follow-up visits by the treating surgeon were performed according to standard hospital routine with the recommended schedule of a visit at 4 weeks, and 3, 6, and 12 months postoperatively.

\section{Adverse Events}

AEs and serious AEs (SAEs) were defined as "any untoward medical occurrence in a subject", and "an AE that led to death, led to serious deterioration in the health of a subject, led to fetal distress, death or congenital abnormality” respectively. All investigators/surgeons classified the AEs in seriousness and its relation to the surgery, MAST approach, and device (unrelated, unlikely, possibly, probably or definitely). For reporting purposes, all AEs were classified into lowest level terms following the medical dictionary for regulatory activities terminology (MedDRA), version 15.1 (V15.1).

\section{Statistical analysis}

For continuous variables, summary statistics were calculated together with the number of missing and non-missing values. For categorical variables, absolute and relative frequencies (based on non-missing values) were provided. The change in each PRO score was calculated for all patients with a valid observation of both the preoperative and four-week score of the PRO in concern. Changes from baseline in PROs at four weeks postoperatively were analyzed (depending on the Shapiro-Wilk test results on normality) using the two-sided $t$-test for paired comparisons and the Wilcoxon signed rank test with a significance level of 0.05. A similar 


\section{Cureus}

strategy was used to evaluate changes between baseline and other follow-up scores, and results are presented as mean $( \pm$ standard deviation $(\mathrm{SD}))$.

\section{Results}

\section{Center contribution}

Contributing surgeons at each center were instructed to contribute 10-30 consecutive degenerative disk disorder (DDD) patients. Once the maximum number of patients had been attained the center participation for new patients was closed, and only follow up activities proceeded for one year.

\section{Patient disposition and baseline demographics}

A total of 252 patients underwent an MIS procedure of which 233 (92\%) patients remained at follow-up for one-year post-surgery. Eleven patients (4.4\%) were lost to follow-up, four withdrew (1.6\%), and four (1.6\%) were explanted resulting in a total of 19 patients who were unavailable for follow-up at one-year post-surgery. Characteristics of the total MIS population were as follows: $56.3 \%$ of patients were female; mean age was $53.8, \pm 11.8$ years; mean body mass index (BMI) was $27.7, \pm 4.6 \mathrm{~kg} / \mathrm{m}^{2}$; mean duration of symptoms resulting in planned surgery was $28.5, \pm 38.2$ months; mean duration of conservative treatment was $20.7, \pm 34.3$ months; and the main indications for surgery were leg pain (52\%), back pain (38.9\%), and neurogenic claudication (9.1\%). Patient demographics are shown in Table 2. Spondylolisthesis (52.8\%), stenosis (71.4\%), and disc pathology (93.7\%) were the most common preoperative degenerative lumbar pathologies. Of the spondylolisthesis cases, $69 \%$ were degenerative, $84 \%$ were Grade 1, and 16\% were Grade 2 according to the Meyerding classification.

Total number of patients

Gender (\% females)

Mean ( $\pm S D)$ age $(y)$

Mean $( \pm S D)$ BMI $\left(\mathrm{kg} / \mathrm{m}^{2}\right)$

Mean $( \pm \mathrm{SD})$ duration of symptoms resulting in planned surgery $(\mathrm{mo})$

Mean $( \pm S D)$ duration of conservative treatment (mo)

Pre-existing conditions relevant to study (\%)

Previous lumbar surgeries (\%)

At target level:

Microdiscectomy open surgery

Microdiscectomy minimally invasive surgery

Decompression (minimally invasive surgery)

TABLE 2: Patient demographics and baseline characteristics 


\section{Cureus}

\section{Surgical procedures performed}

One-level surgery was performed in $83.3 \%$ (210/252) patients; of these, the majority of cases (90.5\%) were interbody fusion at the L4-L5 or L5-S1 levels. 16.7\% (42 patients) underwent twolevel surgery, where fusion was most frequently (73.8\%) performed at L4-S1.

First level surgical approaches varied substantially. Unilateral mini-open approaches were more commonly performed (61.9\%) either without decompression of neural structures (18.3\%) or with unilateral (36.5\%) or bilateral (7.1\%) decompression. Bilateral mini-open approaches (38.1\%) were performed without decompression (5.2\%) or with unilateral (24.6\%) or bilateral (8.3\%) decompression.

A minority of surgeons reported the use of a navigation system (i.e., pre-CT scan (3.6\%), 3D fluoroscopy (1.6\%), image fusion technique (2.4\%), and O-arm imaging (5.6\%) (Table 3). Most fixation techniques were bilateral percutaneous (48.8\%) or bilateral mini-open (39.7\%). For the interbody fusion, most surgeries used an autograft 75.0\% (189/252), while 15.9\% (40/252) used allograft bone bank, and $45.5 \%$ of the autograft were used in conjunction with nanocrystalline hydroxyapatite (86/189).

\section{Mean ( $(\mathrm{SD})$ surgery duration (min)}

One level

Two level

Mean $( \pm S D)$ estimated blood loss $(\mathrm{mL})$

One level

Two level

Blood transfusion, n (\%)

Mean $( \pm S D)$ total fluoroscopy time (sec)

One level

Two level

Navigation system used, n (\%)

Pre-CT

3D fluoroscopy

Image fusion technique

O-arm imaging

Prophylactic antibiotics prescribed, n (\%)
$136.8( \pm 50.4)$

$127.7( \pm 43.5)$

$182.0( \pm 58.3)$

$175.8( \pm 160.1)$

$163.9( \pm 139.7)$

$233.1( \pm 229.0)$

$1(0.4)$

$122( \pm 130.7)$

$115.1( \pm 123.9)$

$154.1( \pm 156.6)$

TABLE 3: Surgery details 


\section{Outcomes}

Primary Outcomes

Across 252 patients, the mean TFA was $1.3 \pm 0.5$ days, the mean TSR was $3.2 \pm 2$ days, and the mean time to discharge was 6.3 days [5].

Secondary Outcomes at One-Year Follow-Up (PRO)

Statistically significant and clinically meaningful changes were reported postoperatively as early as four weeks and were maintained or further improved over one year for each of the PROs assessed. From baseline to one year, there was a 3.3 and 3.8 points improvement in back $(2.9 \pm 2.5$ vs. $6.2 \pm 2.3)$ and leg pain intensity ( $2.2 \pm 2.6$ vs. $5.9 \pm 2.8)$ respectively, $(P<.0001$; Figure 1). Clinical success in reducing back pain was defined as a minimal change in back pain $(\geqslant 30 \%$ improvement from baseline or a change of $\geqslant 1.5$ on a $0-10$ VAS scale) [18] and was achieved at two days after surgery by $46 \%$ and $53 \%$ of patients respectively. This increased throughout the study to more than $70 \%$ and $72 \%$ of patients by one year, respectively. Considering the abovementioned thresholds for leg pain, clinical success was achieved by $69 \%$ and $69 \%$ of patients at two days after surgery, increasing to $73 \%$ and $74 \%$ of patients at one year respectively.

Statistically significant reductions in ODI were reported beginning at four weeks and continued through one year, reaching an improvement in score of $22.4 \% \pm 18.6$ vs. baseline $(45.3 \% \pm 15.3)$, $(P<.0001$; Figure 2). Minimally important changes $(\geqslant 30 \%$ improvement from baseline or improvement in ODI score of $\geqslant 10$ ) in disability were achieved by more than $41 \%$ and $51 \%$ of patients respectively at four weeks which increased to more than $70 \%$ and $77 \%$, respectively by one year. 


\section{Cureus}

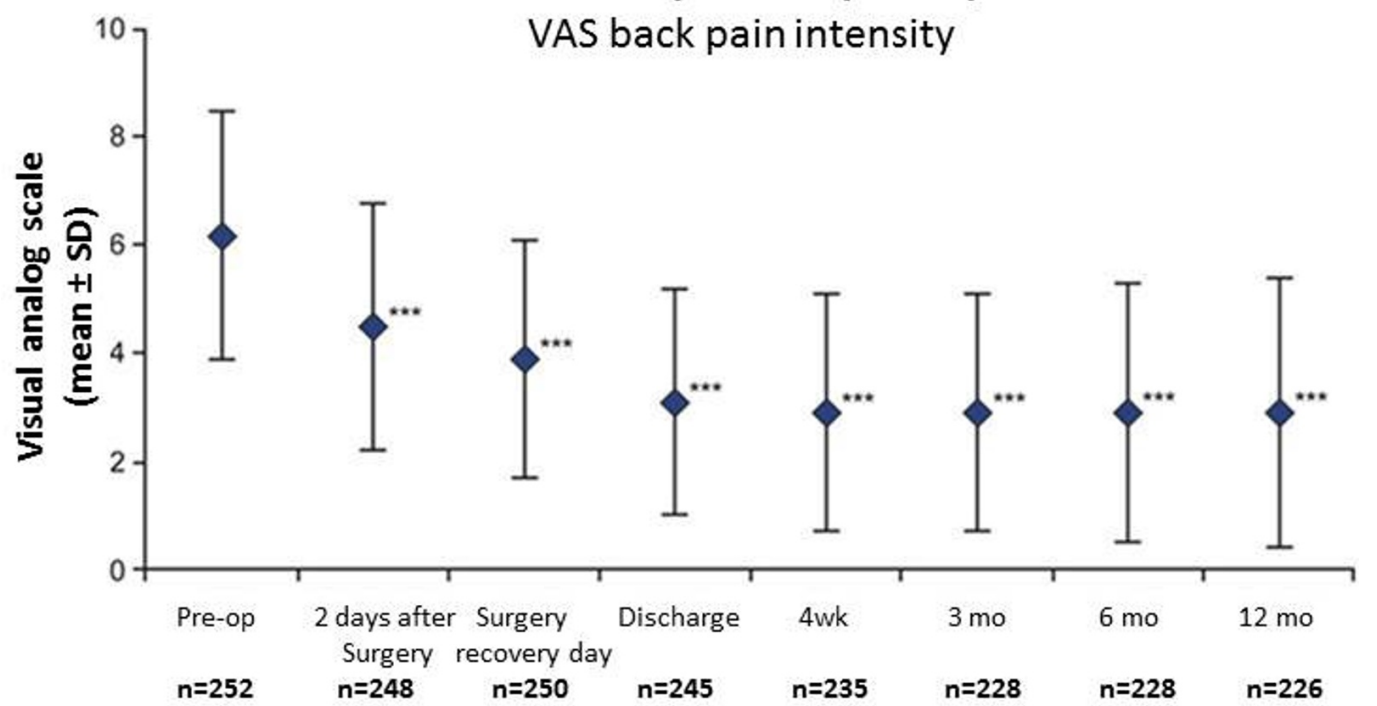

b

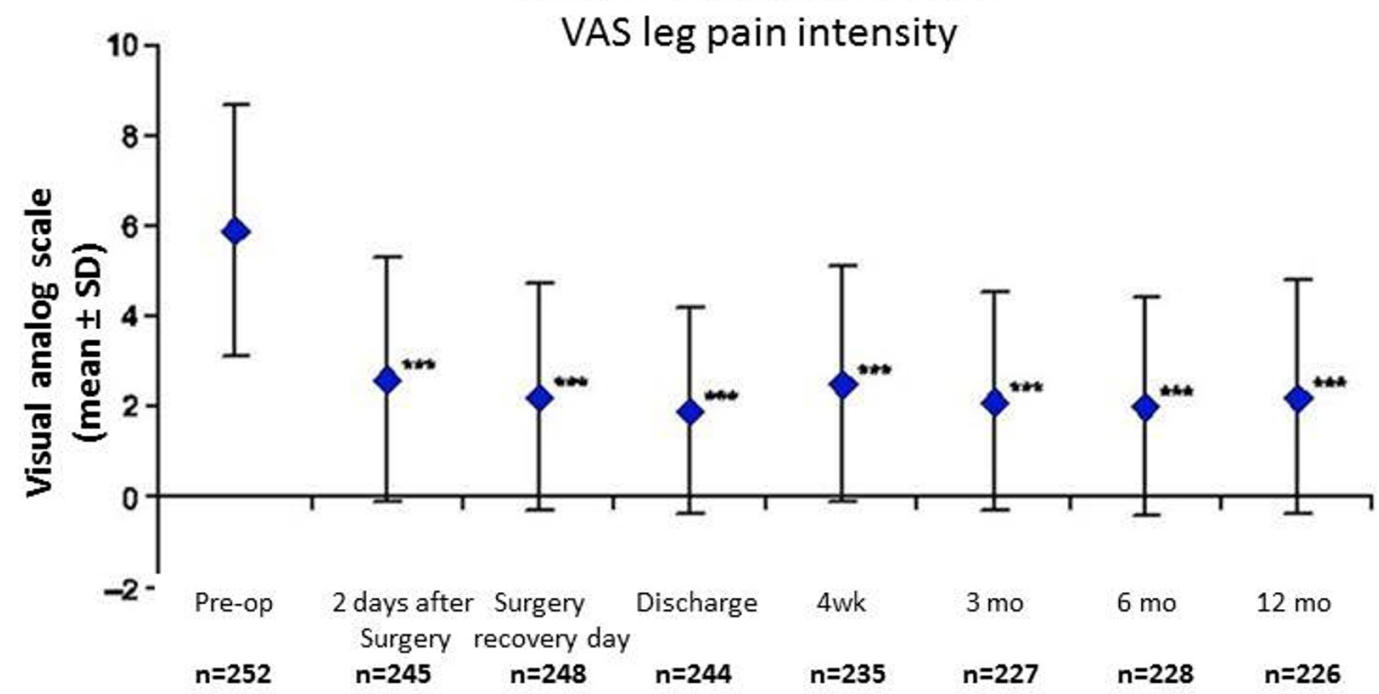

FIGURE 1: Improvement of back and leg pain.

Back (a) and leg (b) pain intensity scores reported preoperatively and postoperatively on a $10 \mathrm{~cm}$ visual analog scale (VAS) where $0=$ minimal pain intensity or pain frequency and $10=$ maximal pain intensity or pain frequency (total population, $\mathrm{n}=252$ ). ${ }^{* * *} P<.0001$ for difference between preoperative (back $6.2 \pm 2.3$, leg $5.9 \pm 2.8$ ) and postoperative scores at two days (back $4.5 \pm 2.3$, leg $2.6 \pm 2.7$ ), surgery recovery day (back $3.9 \pm 2.2$, leg $2.2 \pm 2.5$ ), discharge (back $3.1 \pm 2.1$, leg 1.9 \pm 2.3 ), four weeks (back $2.9 \pm 2.2$, leg $2.5 \pm 2.6$ ), three months (back $2.9 \pm 2.2$, leg $2.1 \pm 2.4$ ), six months (back $2.9 \pm 2.4$, leg $2.0 \pm 2.4$ ), and twelve months (back $2.9 \pm 2.5$, leg $2.2 \pm 2.6$ ). 


\section{Cureus}

MAST population $(n=252)$

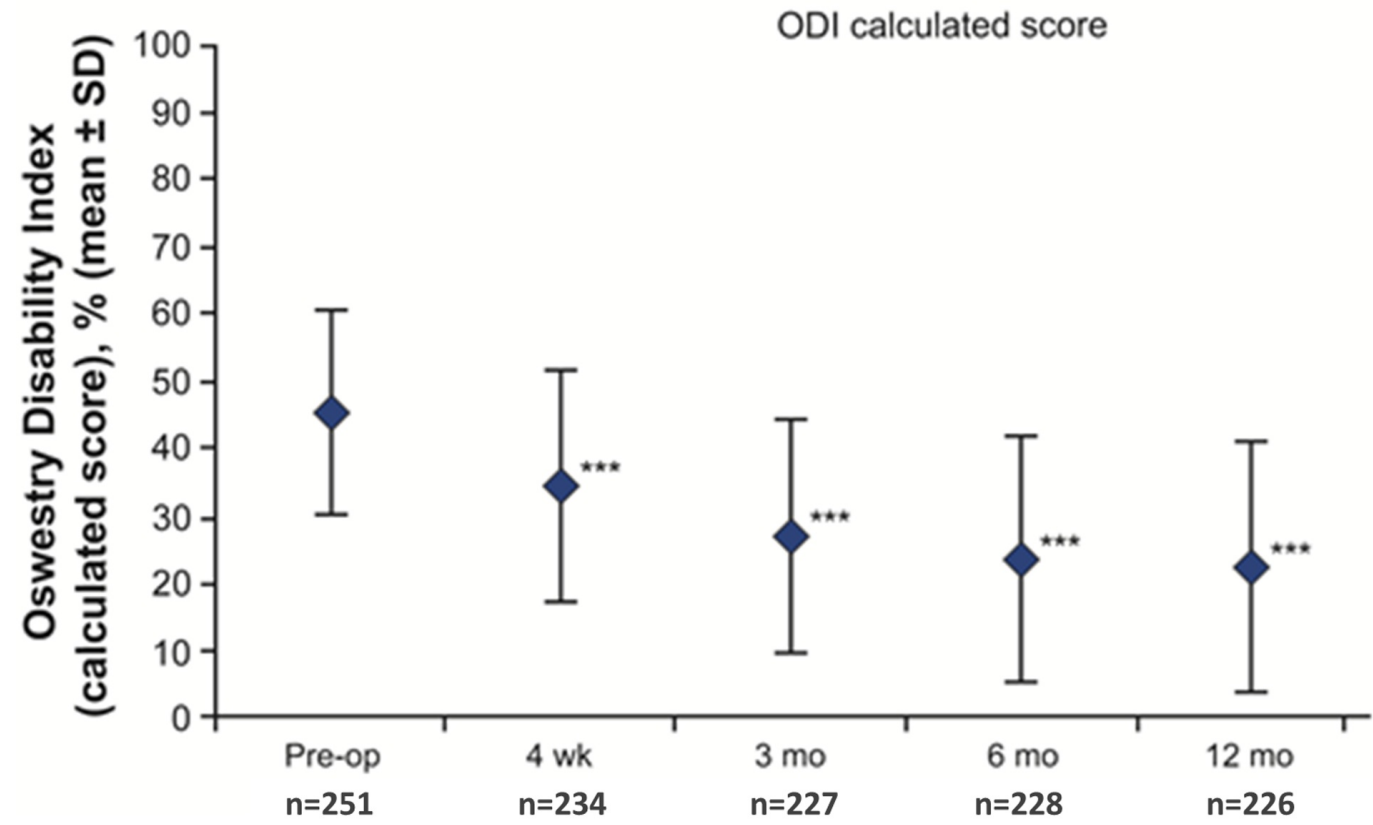

FIGURE 2: Improvement of patient disability.

Oswestry disability index (ODI) scores (calculated in \%) reported preoperatively and at four weeks and three, six, and twelve months postoperatively on a $0 \%$ to $100 \%$ scale, where $0 \%=$ minimal disability and $100 \%=$ maximal disability (total population, $\mathrm{n}=252)$. ${ }^{* * *} P<.0001$.

Improvement in EQ-VAS scores vs. preoperative scores began at four weeks (12.0 points; $P$ $<.0001)$ and reached 18.0 points at one year $(P<.0001)$. At one year, the proportion of patients who reported no health-related problems increased from the preoperative values in each of the five individual EQ-5D domains: mobility (57.2\% vs. $11.1 \%)$, self-care $(75.4 \%$ vs. $54.3 \%)$, usual activities (56.7\% vs. 9.6\%), pain/discomfort (30.3\% vs. $1.4 \%$ ), and anxiety/depression (69.6\% vs. 49.5\%) (Figure 3). For each domain, improvements were apparent as early as four weeks and continued to increase through one year. The EQ-5D index improved from a mean of $0.34 \pm 0.32$ preoperatively to $0.71 \pm 0.28$ at one-year follow-up. 


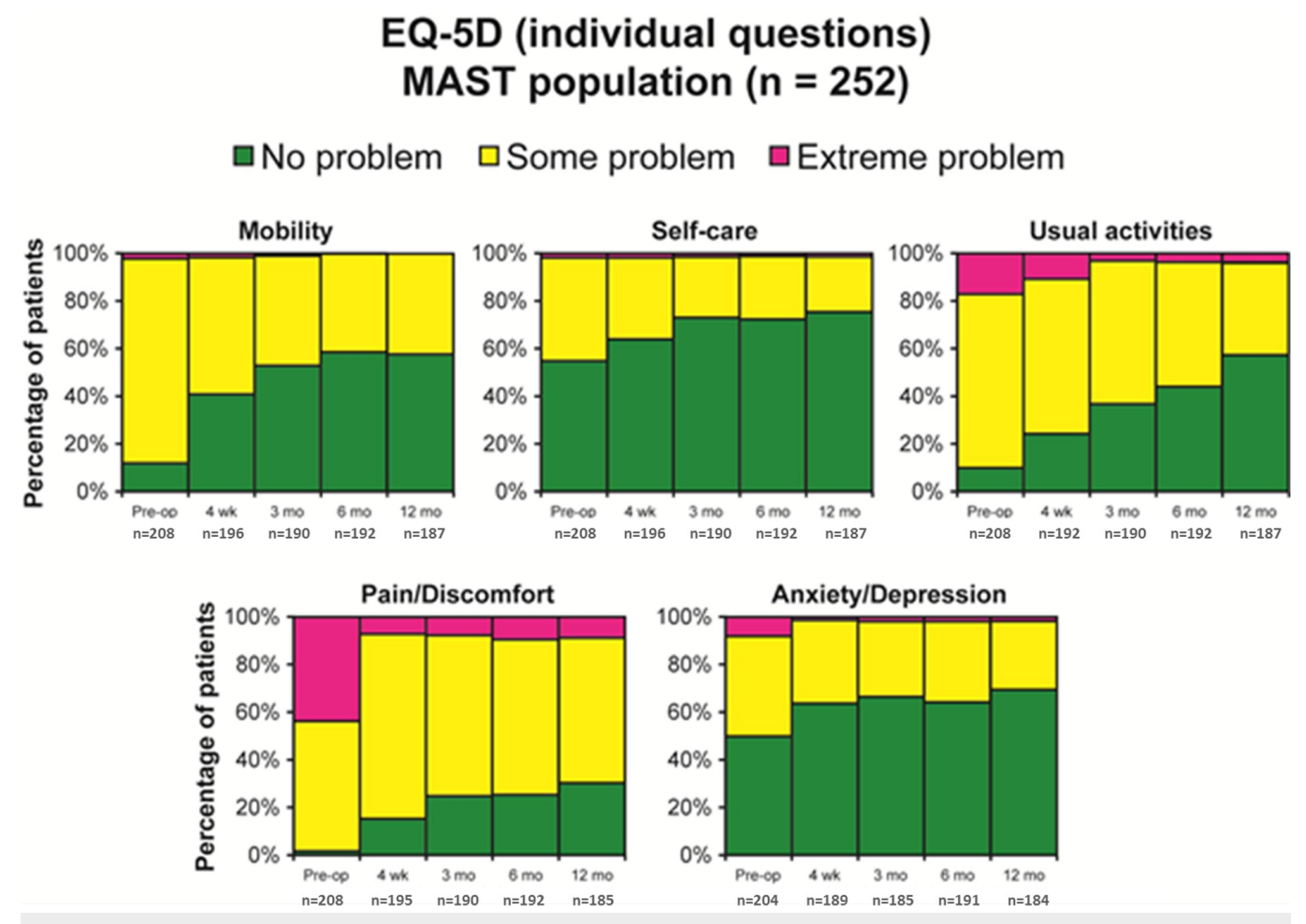

FIGURE 3: Overall amelioration in quality of life.

Percentages of patients who reported on five EQ-5D domains preoperatively vs. four weeks, three months, six months and twelve months.

Clinical and Radiological Surgery Outcomes

Assessment of fusion was not standard of care at all sites, and not all levels treated were assessed for fusion success. One hundred thirty one-level and 24 two-level patients who were assessed for fusion as per protocol at one year; the fusion rates were $90.8 \%$ (118/130) and 89.6\% (43/48) respectively. Some sites assessed fusion at one year, but used different site-specific criteria than those set out in the protocol of this study; an additional 14 patients (11 one-level and 3 two-level) were assessed using non-protocol defined criteria had fusion rates of $90.9 \%$ (10/11 levels) and 100\% (6/6 levels) respectively.

The rate of additional lumbar spinal surgeries was low at $2.8 \%(7 / 252)$ in seven patients at six centers. Of these seven additional surgeries, three (1.2\%) were reoperations (at the same level-loss of pedicle screw fixation causing recurrent spondylolisthesis and left leg radiculopathy, revision of right pedicle screw S1, vertebral body collapse of upper target vertebra), and four (1.6\%) were performed at an adjacent level (Disc herniation on upper adjacent level with extrusion on the right side, pedicle screw dislocation because of osteoporotic bone and trauma, intraspinal hemorrhage at one and two levels above the previously operated levels, and preexisting lower adjacent level of DDD).

Likely reflective of local clinical practice, fewer than half $(45 \%, 102 / 226)$ of the patients were receiving rehabilitation at three months. Assessed by the surgeon at one year, the satisfaction with the results of treatment after the surgery was $81 \%$ (177/218), while $78 \%(171 / 218)$ indicated to be helped by the treatment as expected, and $82 \%(178 / 218)$ indicated to have the same treatment again for the same condition. At one year, the surgeons indicated that $85.8 \%$ of the patients were completely improved $(28 \%, 61 / 218)$, much improved $(41 \%, 89 / 218)$, or slightly 


\section{Cureus}

improved 17\% (37/218) with regard to their back pain recovery compared to preoperative levels.

Ability to return to work (full- and part-time work) increased postoperatively among professional workers which composed of $57 \%$ of the total patient population (143/252). At the preoperative visit, 55.2\% (79/143) were working, while at one year postoperatively, this number climbed to $70.3 \%(90 / 128)$. The rate of professional workers being on paid leave/disability because of back problems was halved from pre-op (57/143, 39.9\%) to one year $(22 / 128,17.2 \%)$.

Even among patients who were still taking lumbar spine pain medications (68.7\% pre-op vs $41.5 \%$ at one year), the frequency and potency of pain medication use decreased over the one year after surgery vs. preoperative conditions (Figure 4).

MAST population $(n=252)$

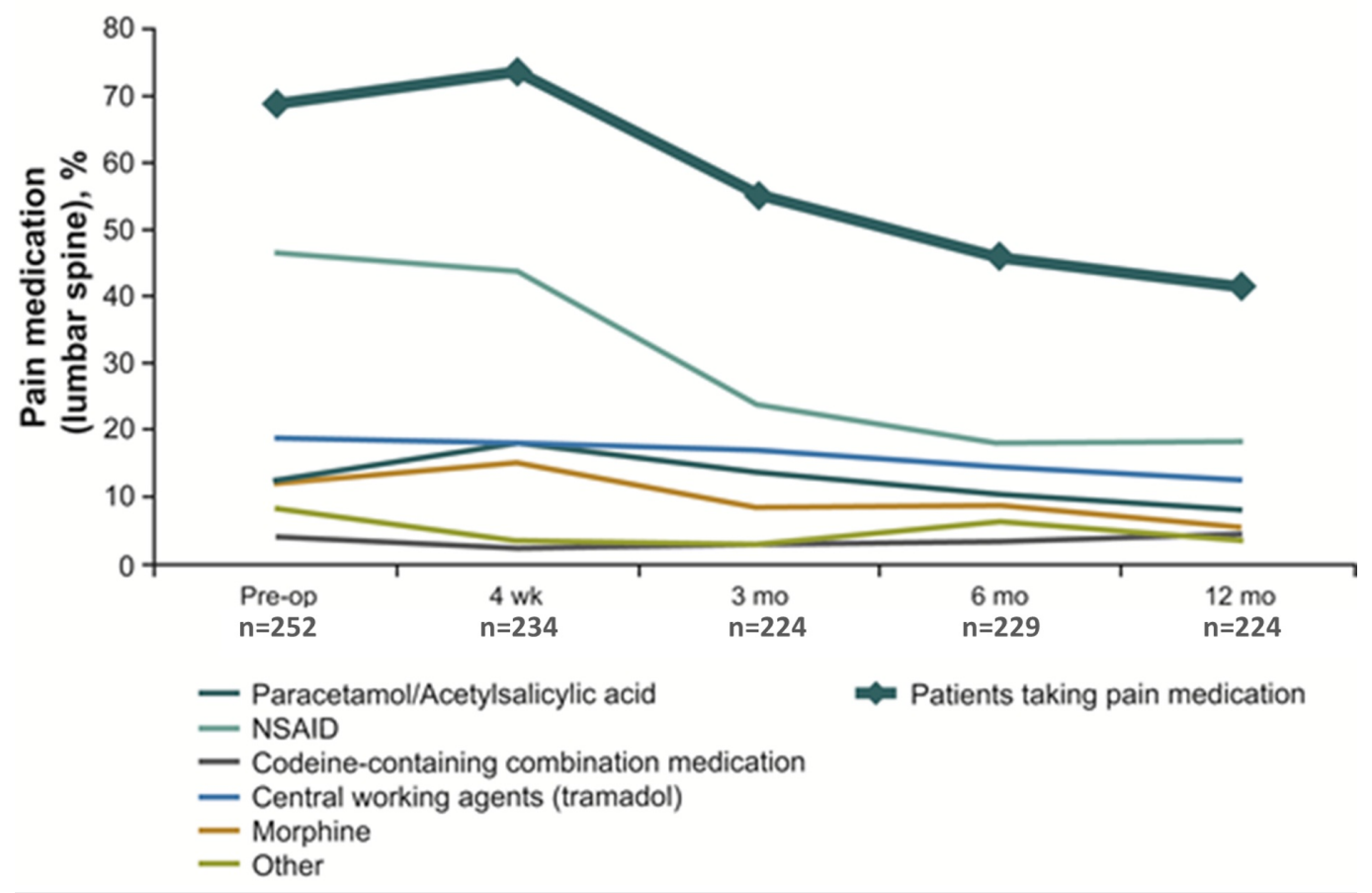

\section{FIGURE 4: Decreased frequency and potency of pain} medication for lumbar spine in the past week.

Percentages may not equal $100 \%$ because combinations of medications may have been used by the same patient.

Adverse Events

Throughout the study, 50 AEs in 39 patients were considered by the investigator to be related to surgery, MAST, or device. Nine of these were SAEs (acute allergic reaction, postoperative confusion, leg pain, back pain, lumbar disc herniation, spinal hematoma, and urosepsis). Out of these 50 AEs, 3 AEs $(0.79 \%, 2 / 252)$ and 1 SAE (increased leg pain) were considered related to the minimally invasive approach (Table 4 ); there was one superficial incision site abscess related to surgery $(0.4 \%)$, and there were no deep wound infections among the 252 patients. 


\section{Cureus}

\begin{tabular}{|c|c|c|}
\hline $\begin{array}{l}\text { MedDRA code low level } \\
\text { terms }\end{array}$ & $\begin{array}{l}\text { Count of MAST }{ }^{\mathrm{TM}} \\
\text { related }\end{array}$ & $\begin{array}{l}\text { Count of total related to device, surgery, or MAST }{ }^{\mathrm{TM}} \\
\text { (serious event) }\end{array}$ \\
\hline Acute allergic reaction & 0 & $1(1)^{\star}$ \\
\hline Back pain & 0 & $7(1)^{*}$ \\
\hline Confusion postoperative & 0 & $1(1)^{*}$ \\
\hline Dural tear & 0 & 4 \\
\hline Fever & 0 & 2 \\
\hline Hypoesthesia & 0 & 3 \\
\hline Implant site seroma & 0 & 2 \\
\hline Implant site abcess & 0 & 1 \\
\hline Leg pain & $3(1)^{*}$ & $10(3)^{*}$ \\
\hline Lumbar disc herniation & 0 & $1(1)^{*}$ \\
\hline Lumbar radiculopathy & 0 & 3 \\
\hline Nausea & 0 & 4 \\
\hline Sacro-iliac pain & 0 & 4 \\
\hline Spinal hematoma & 0 & $1(1)^{*}$ \\
\hline Urinary tract infection & 0 & 3 \\
\hline Urosepsis & 0 & $1(1)^{*}$ \\
\hline Vertigo & 0 & 1 \\
\hline Vomiting & 0 & 1 \\
\hline Total & $3(1)$ & $50(9)$ \\
\hline
\end{tabular}

TABLE 4: Adverse events in total population over twelve months

* Parentheses indicate a serious adverse event.

\section{Discussion}

We reported the one-year results of a pragmatic, multicenter, prospective, monitored study using minimally invasive fusion strategies in the daily surgical practice setting in 19 countries. Our results indicate that MILIF outcomes are in line with other reported surgical studies for the spine and provide valuable insights into the management of DLD in the usual clinical practice. This observational study did not add to the patient burden of illness and has gathered a large network of surgeons who have cooperated to collect a comprehensive set of outcomes including an assessment of long-term outcomes through the achievement of high levels of one-year 
follow-up (92\%).

Short surgery duration and low blood loss achieved with MILIF at four weeks after surgery were beneficial for patients as they aided in faster recovery [5]. These improved clinical results with low complication and reoperation rates were maintained over the one year of the study. Statistically significant and consistent results in all assessed PROs (VAS back and leg pain, ODI, and EQ-5D) were seen from two days after surgery and were maintained throughout the oneyear study indicating reduced pain, better mobility, and improvements in quality of life. Clinical success [18] was demonstrated in the rapid and persistent improvement in pain and disability, which started as early as two days after surgery and was maintained or improved throughout the one-year study. Reflective of the improvements in pain and disability, high levels of patient satisfaction with surgery (81\%) and reductions in both frequency and potency of pain medications were observed vs. baseline over the one year after surgery. In addition, low levels of formal rehabilitation at one year have been reported (27.0\%). Reduction in the use of pain medications after MIS are also consistent with previous reports [11, 14, 19-22]. In a retrospective study, narcotic use decreased from $100 \%$ before MIS to $31 \%$ by six months postoperatively [21]. Perioperative morphine use was significantly reduced after MIS TLIF vs. open surgery [11] and patients who received MIS had a significantly faster narcotic independence [22].

Patient well-being was indicated by an improvement in their disability and their ability to return to work. At the one-year postoperative time point, the evaluation of work status included both patients who had and had not been working prior to surgery. At one year, 70.3\% (90/128) of patients were working compared with $55.2 \%(43 / 79)$ at the preoperative assessment. Moreover, the number of patients on paid leave/disability was halved in one year (39.9\% down to $17.2 \%)$. Improvements in the ability to return to work in this study are in agreement with published reports. In a long-term prospective study, more patients were working (>68\%) at two to six years after open or laparoscopic surgery for DDD than had worked preoperatively (52\%) [19]. Adogwa et al., 2011 [23] reported a faster return to work (8.5 weeks vs. 17.1 weeks) for patients treated with MIS vs. open surgery, while Parker 2012 described 90\% of patients who received MIS TLIF returning to work postoperatively vs. $80 \%$ of patients treated with open surgery. In another study, $97 \%$ of patients who were working prior to MIS TLIF were able to return to work postoperatively [21].

The range of fusion success rate (89.6\%-100.0\%), regardless of how it was measured, was similar to that of published literature using minimally invasive and open techniques $[3-4,7,11$ $12,20,22]$. The monitoring of our study allowed us to closely follow up the safety of the device and procedures. The majority of surgical procedures in this study was achieved with minimal short- or long-term complications. Throughout the study, 3 out of 50 (1.19\%) AEs were considered specifically MIS-related by the surgeons; out of these three AEs, one was a serious event (severe leg pain). The majority of AEs associated with MIS reported in the literature are site infections. We reported a $0.4 \%$ infection rate at four weeks and no deep surgical site infections were observed at one-year follow-up. A comparable infection rate of $0.22 \%$ was observed in retrospective studies such as a large cohort of patients $(\mathrm{N}=1274)$ [24] who received MILIF. In another retrospective study ( $\mathrm{N}=5170)$, surgical site infections were $4.6 \%$ vs. $7.0 \%(P$ $=.037$ ) for two-level MILIF compared with open surgery, and $4.5 \%$ vs. $4.8 \%$ ( $P=.77)$ for one-level MILIF vs. open surgery [25], $0.6 \%$ vs. $4 \%, \mathrm{p}=0.0005, \mathrm{n}=362$ TLIF, $\mathrm{n}=1133$ open TLIF [22].

Reflective of clinical practice, the majority of surgeries performed in this study included unilateral decompression, and a minor number of surgeries were performed with the use of a navigation system. However, the low use of navigation systems may be the cause for higher radiation exposure (122.0 \pm 130.7$)$ compared to other studies ( $38.7 \mathrm{~s}, 71 \mathrm{~s}, 101 \mathrm{~s})$ [26-28]. In order to minimize the exposure, it is recommended that surgeons use intermittent fluoroscopy and keep an adequate distance from the X-ray tube [28] and navigation systems 
[29]. It is important to note that the majority of procedures $(75 \%, 189 / 252)$ were performed using autografting, often in conjunction with an osteoconductive synthetic bone paste. The most common graft substitute used in combination with autograft was nanocrystal hydroxyapatite $(45.5 \%, 86 / 189)$. However, data was only taken from those centers that proactively reported the types of graft substitutes used and may not be reflective of the entire population.

\section{Limitations of the study}

The lack of a direct comparison of outcomes for a controlled group (MIS and open surgery) as seen in randomized controlled trials (RCTs) was a limitation of the study. Our data is reflective of the usual clinical practice of MIS around the world and collected from a non-selected patient population by investigators who were allowed to choose the management strategies normally available to them in their standard practice. This study shows that MILIF is a feasible treatment for DLD, provides valuable insights on how practitioners apply MILIF in their practice, and will facilitate the design of future studies and identify specific indications or patient subpopulations for MILIF. The study fulfills some of the criteria for observational studies [30] such as a large and diverse population of patients in real-world settings, allows for the ability to detect minor treatment effects or rare complications over one year, provides insight into the clinical context in which multiple or complex therapies are delivered and is relatively inexpensive and can be performed rapidly. However, the study does not fulfill the criteria of confounding or selection bias of patients despite the defined inclusion criteria as it is an observational study without a control. It may very well be that the surgeons only selected in their view the 'best' patient for the surgical procedure as in 'real clinical practice'.

\section{Successes of the study}

The study assembled a large group of experienced and willing surgeons to participate in the study. The study was monitored by an independent agency, and the study protocol was decided well in advance. Research nurses were available for data quality and data gathering as little data is missing, and there is a low dropout rate as only 19 patients of 252 dropped out over the course of one year. The short-term results over one year are in line with the literature of other spinal surgeries for DDD. Important data for patients and surgeons was gathered and useful data for the clinical community has been presented. Future studies will have to support or refute the outcomes of this study.

\section{Conclusions}

This is the first pragmatic, international, multicenter, prospective, monitored outcomes study evaluating MILIF to treat DLD under usual surgical practice and to monitor progress over the course of one year. Statistically significant and consistent results were observed for all PROs; patients achieved early mobility, decreased leg and back pain, decreased disability, high patient satisfaction, and more were able to work postoperatively than had been working prior to surgery. This study shows that improvements which were observed four weeks post-surgery were maintained or further improved up to one year with complications. The results suggest that MILIF is an effective, feasible, and safe treatment for DLD in a broad patient population under usual clinical conditions. Further investigation in a larger patient population will provide insights on the long-term benefits of MILIF.

\section{Additional Information}

\section{Disclosures}

Human subjects: Consent was obtained by all participants in this study. Animal subjects: All authors have confirmed that this study did not involve animal subjects or tissue. Conflicts of 
interest: In compliance with the ICMJE uniform disclosure form, all authors declare the following: Payment/services info: Grant from Medtronic for my institution for 15000 dollars. Fees for participating in review activities such as data monitoring boards, statistical analysis, end point committees etc. from Medtronic for 2000 dollars. Financial relationships: Joerg Franke declare(s) employment from Paradigm. Speaking/teaching arrangements for 15000 dollars. Joerg Franke declare(s) personal fees from Zimmer. Speaking/teaching arrangements for 10000 dollars. Joerg Franke declare(s) personal fees from Philipps. Speaking/teaching arrangements for 2500 dollars. Joerg Franke declare(s) Money to institution from Baxter. Research support for 50000 dollar. Joerg Franke declare(s) Money to Institution from Paradigm Spine. Research support for 25000 dollar. Arkadiusz Kosmala, Wout Rosenberg, Frederic Martens, Khai Lam, Peter Durny declare(s) Did not receive any payment or service from a 3rd party for any aspect of the submitted work. from Nothing to declare. Has no financial interests with entities as described in the ICMJE instructions. Zvi Lidar declare(s) For participating in review activities such as data monitoring boards, statistical analysis, end point committees etc. for 3000 dollars from Tel Aviv Medical Center. Fees to my institution. Joerg Franke, Neil Manson, David Buzek, Ulrich Hubbe, Roberto Assietti, Kai-Michael Scheufler, Wolfgang Senker declare(s) a grant, personal fees, employment and Money to institution (Scientific advisory board/other office) Research support for 15000 dollar from Medtronic. Consulting for 5000 dollars (J.F) Speaking/teaching arrangements for 30000 dollars (J.F) Scientific advisory board/other office for 2000 dollar (J.F) Speaking/teaching arrangements less than 10000 dollar/year (N.M) Scientific advisory board, non-financial and financial support less than 10000 dollar (N.M) Research support, Employment, research co-ordinator salary (N.M) Consulting Fees, 15800 dollar (D.B) Consulting fees (U.H) Speaking/teaching arrangements (U.H) Trips/Travel (U.H) Research support for 500 dollars. (U.H) Speaking/teaching arrangement for 10000 dollars (R.A) Personal fees for travel and meetings for 3500 dollars (K.S) Consulting fees for 3000 dollars (K.S) Speaking/teaching arrangements for 15000 dollars (K.S) Travel to instructional locations for 1000 dollars (W.S) Speaking/teaching arrangements for 500 dollars (W.S). Paulo Pereira declare(s) personal fees from Depuy. Consulting fees from Depuy for 1500 dollars. Other relationships: All authors have declared that there are no other relationships or activities that could appear to have influenced the submitted work.

\section{Acknowledgements}

The authors wish to thank the remaining MASTERS-D investigators for their contribution to this study: Prof. Dr. Salvador Fuster, Spain; Dr. Walter Richter, Germany; Dr. Marcus Schröder, The Netherlands; Prof. Pawel Sloniewski, Poland; Dr. Vassilios Vougioukas, Greece; the Medtronic clinical study managers Christel Van Dam and Dr Cristina Faria for conducting the MASTERS-D study; medical affairs managers Dr Alexander Cristea, Dr. Kirstin Demesmaeker, scientific communications specialist Dr Mayra Mori for technical review of the manuscript. The authors would like to thank Bert Parmet and Jacques Janssen of Cromsource for performing the statistical analysis and Carol Berry and Pippa Clarke of Quintiles for medical writing and editorial assistance, which were both funded by Medtronic.

\section{References}

1. Guyer RD, Foley KT, Phillips FM, Ball PA: Minimally invasive fusion: summary statement. Spine. 2003, 28:S44. 10.1097/00007632-200308011-00008

2. McAfee PC, Phillips FM, Andersson G, et al.: Minimally invasive spine surgery. Spine. 2010, 35:S271-S273. 10.1097/BRS.0b013e31820250a2

3. Karikari IO, Isaacs RE: Minimally invasive transforaminal lumbar interbody fusion: a review of techniques and outcomes. Spine. 2010, 35:S294-S301. 10.1097/BRS.0b013e3182022ddc

4. Chaudhary KS, Groff MW: Minimally invasive transforaminal lumbar interbody fusion for degenerative spine. Tech Orthop. 2011, 26:146-55. 10.1097/BTO.0b013e31822ce25d

5. Pereira P, Buzek D, Franke J, et al.: Surgical data and early postoperative outcomes after minimally invasive lumbar interbody fusion: results of a prospective, multicenter, 
observational data-monitored study. PLoS One. 2015, 10:e0122312.

10.1371/journal.pone.0122312

6. Etame AB, Wang AC, Than KD, Park P: Clinical and radiographic outcomes after minimally invasive transforaminal lumbar interbody fusion. SAS J. 2010, 4:47-53.

10.1016/j.esas.2010.03.002

7. Park Y, Ha JW: Comparison of one-level posterior lumbar interbody fusion performed with a minimally invasive approach or a traditional open approach. Spine. 2007, 32:537-543. 10.1097/01.brs.0000256473.49791.f4

8. Rampersaud YR, Gray R, Lewis SJ, Massicotte EM, Fehlings MG: Cost-utility analysis of posterior minimally invasive fusion compared with conventional open fusion for lumbar spondylolisthesis. SAS J. 2011, 5:29-35. 10.1016/j.esas.2011.02.001

9. Schizas C, Tzinieris N, Tsiridis E, Kosmopoulos V: Minimally invasive versus open transforaminal lumbar interbody fusion: evaluating initial experience. Int Orthop. 2009, 33:1683-1688. 10.1007/s00264-008-0687-8

10. Tian NF, Wu YS, Zhang XL, Xu HZ, Chi YL, Mao FM: Minimally invasive versus open transforaminal lumbar interbody fusion: a meta-analysis based on the current evidence. Eur Spine J. 2013, 22:1741-1749. 10.1007/s00586-013-2747-z

11. Peng CW, Yue WM, Poh SY, Yeo W, Tan SB: Clinical and radiological outcomes of minimally invasive versus open transforaminal lumbar interbody fusion. Spine. 2009, 34:1385-1389. 10.1097/BRS.0b013e3181a4e3be

12. Villavicencio AT, Burneikiene S, Roeca CM, Nelson EL, Mason A: Minimally invasive versus open transforaminal lumbar interbody fusion. Surg Neurol Int. 2010, 1:12.

13. Lee JC, Jang HD, Shin BJ: Learning curve and clinical outcomes of minimally invasive transforaminal lumbar interbody fusion: our experience in 86 consecutive cases. Spine. 2012, 37:1548-1557. 10.1097/BRS.0b013e318252d44b

14. Scheufler KM, Dohmen H, Vougioukas VI: Percutaneous transforaminal lumbar interbody fusion for the treatment of degenerative lumbar instability. Neurosurgery. 2007, 60:203-212. 10.1227/01.NEU.0000255388.03088.B7

15. Chapman CR, Casey KL, Dubner R, Foley KM, Gracely RH, Reading AE: Pain measurement: an overview. Pain. 1985, 22:1-31. 10.1016/0304-3959(85)90145-9

16. Fairbank JC, Couper J, Davies JB, O'Brien JP: The Oswestry low back pain disability questionnaire. Physiotherapy. 1980, 66:271-273.

17. Coons SJ, Rao S, Keininger DL, Hays RD: A comparative review of generic quality-of-life instruments. Pharmacoeconomics. 2000, 17:13-35. 10.2165/00019053-200017010-00002

18. Ostelo RW, Deyo RA, Stratford P, et al.: Interpreting change scores for pain and functional status in low back pain: towards international consensus regarding minimal important change. Spine. 2008, 33:90-94. 10.1097/BRS.0b013e31815e3a10

19. Burkus JK, Gornet MF, Schuler TC, Kleeman TJ, Zdeblick TA: Six-year outcomes of anterior lumbar interbody arthrodesis with use of interbody fusion cages and recombinant human bone morphogenetic protein-2. J Bone Joint Surg Am. 2009, 91:1181-1189. 10.2106/JBJS.G.01485

20. Schwender JD, Holly LT, Rouben DP, Foley KT: Minimally invasive transforaminal lumbar interbody fusion (TLIF): technical feasibility and initial results. J Spinal Disord Tech. 2005, 18:S1-S6. 10.1097/01.bsd.0000132291.50455.d0

21. Rouben D, Casnellie M, Ferguson M: Long-term durability of minimal invasive posterior transforaminal lumbar interbody fusion: a clinical and radiographic follow-up. J Spinal Disord Tech. 2011, 24:288-296. 10.1097/BSD.0b013e3181f9a60a

22. Parker SL, Lerner J, McGirt MJ: Effect of minimally invasive technique on return to work and narcotic use following transforaminal lumbar inter-body fusion: a review. Prof Case Manag. 2012, 17:229-235. 10.1097/ncm.0b013e3182529c05

23. Adogwa O, Parker SL, Bydon A, Cheng J, McGirt MJ: Comparative effectiveness of minimally invasive versus open transforaminal lumbar interbody fusion: 2-year assessment of narcotic use, return to work, disability, and quality of life. J Spinal Disord Tech. 2011, 24:479-484.

24. O'Toole JE, Eichholz KM, Fessler RG: Surgical site infection rates after minimally invasive spinal surgery. J Neurosurg Spine. 2009, 11:471-476. 10.3171/2009.5.SPINE08633

25. McGirt MJ, Parker SL, Lerner J, Engelhart L, Knight T, Wang MY: Comparative analysis of perioperative surgical site infection after minimally invasive versus open posterior/transforaminal lumbar interbody fusion: analysis of hospital billing and discharge 


\section{Cureus}

data from 5170 patients. J Neurosurg Spine. 2011, 14:771-778. 10.3171/2011.1.SPINE10571

26. Funao H, Ishii K, Momoshima S, et al.: Surgeons' exposure to radiation in single- and multilevel minimally invasive transforaminal lumbar interbody fusion; a prospective study. PLoS One. 2014, 9:e95233. 10.1371/journal.pone.0095233

27. Bindal RK, Glaze S, Ognoskie M, Tunner V, Malone R, Ghosh S: Surgeon and patient radiation exposure in minimally invasive transforaminal lumbar interbody fusion. J Neurosurg Spine. 2008, 9:570-573. 10.3171/spi.2008.4.08182

28. Wang J, Zhou Y, Zhang ZF, Li CQ, Zheng WJ, Liu J: Minimally invasive or open transforaminal lumbar interbody fusion as revision surgery for patients previously treated by open discectomy and decompression of the lumbar spine. Eur Spine J. 2011, 20:623-628. 10.1007/s00586-010-1578-4

29. Bandela JR, Jacob RP, Arreola M, Griglock TM, Bova F, Yang M: Use of CT-based intraoperative spinal navigation: management of radiation exposure to operator, staff, and patients. World Neurosurg. 2013, 79:390-394. 10.1016/j.wneu.2011.05.019

30. Nallamothu BK, Hayward RA, Bates ER: Beyond the randomized clinical trial: the role of effectiveness studies in evaluating cardiovascular therapies. Circulation. 2008, 118:12941303. 10.1161/CIRCULATIONAHA.107.703579 